\title{
The existence of an endoscopically observable capillary plexus in the deep colonic
} mucosa

Colonoscopy allows microvascular observation on the luminal surface; however, the precise depth of the blood vessels being observed is unknown. We successfully generated $3 \mathrm{D}$ reconstructed images of the microvessels of the crypts and associated mucosa from tissue slides using Synapse VINCENT [1,2]. Colonoscopic images of a normal colon were compared with the histological preparation of the colon microvasculature to clarify the depth of the vascular structures being observed by colonoscopy.

We present colonoscopic images of a 51-year-old man with normal mucosa; he had provided written informed consent. The sigmoid colon was observed with the white light and blue laser imaging (BLI) modes of the LASEREO endoscope system of an EC-L590ZW colonoscope (Fujifilm, Tokyo, Japan).

- Video 1 shows the two plexuses of the colonic mucosal capillary network and their connections. One plexus was present at the subepithelial level; the other was at the crypt bottom, just above the muscularis mucosa. The two plexuses were connected via capillaries that ran along the crypts. We reconstructed a $3 \mathrm{D}$ histological image from the 2D tissue section images acquired by digital microscopy and compared this with the endoscopy images ( $\mathbf{F i g . 1}$ ); the capillary plexus in the deep mucosa could be observed with both white light and BLI at low magnification.

It is believed that the arteries that enter the colon wall form a submucosal plexus with repeated bifurcations, and then rise through the muscularis mucosa to form a polygonal capillary plexus below the epithelium [3, 4]. However, our results revealed that the colonic mucosa had capillary plexuses not only at the subepithelial level but also at the crypt bottom level. By providing crypt stem cells with oxygen and nutrients, a deep mucosal capillary plexus can be formed. This is the first report showing the existence of an endo-

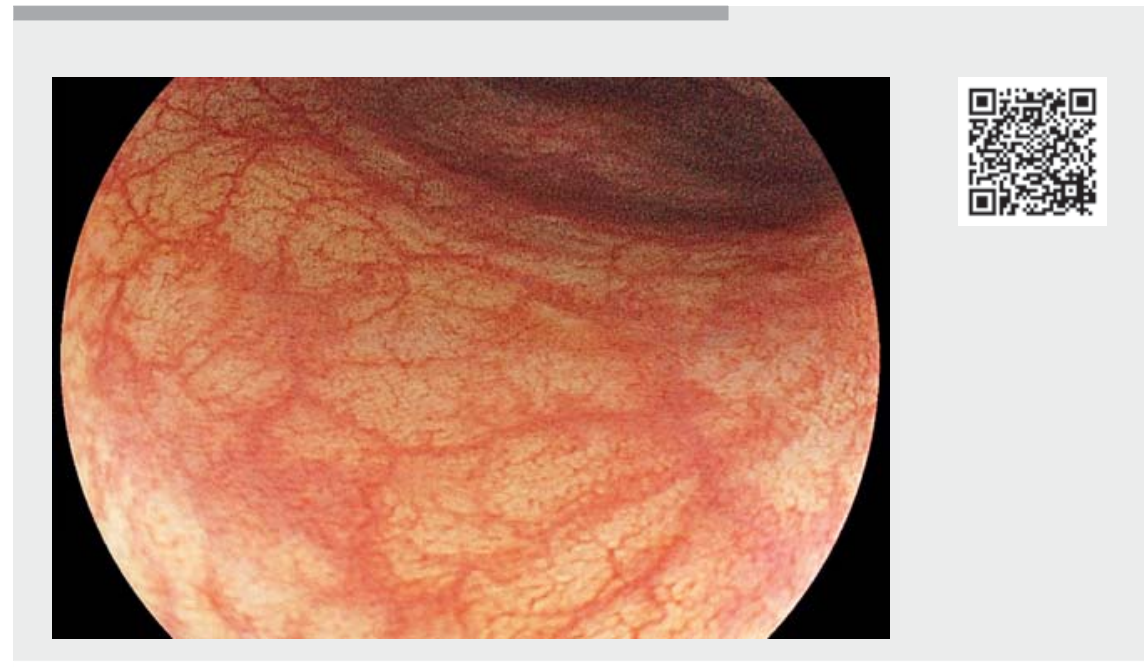

$\nabla$ Video 1 The creation of 3D reconstructed images of the crypts and microvessels extracted from serial sections of normal colonic mucosal tissue using the Synapse VINCENT software program (Fujifilm Co., Tokyo, Japan).
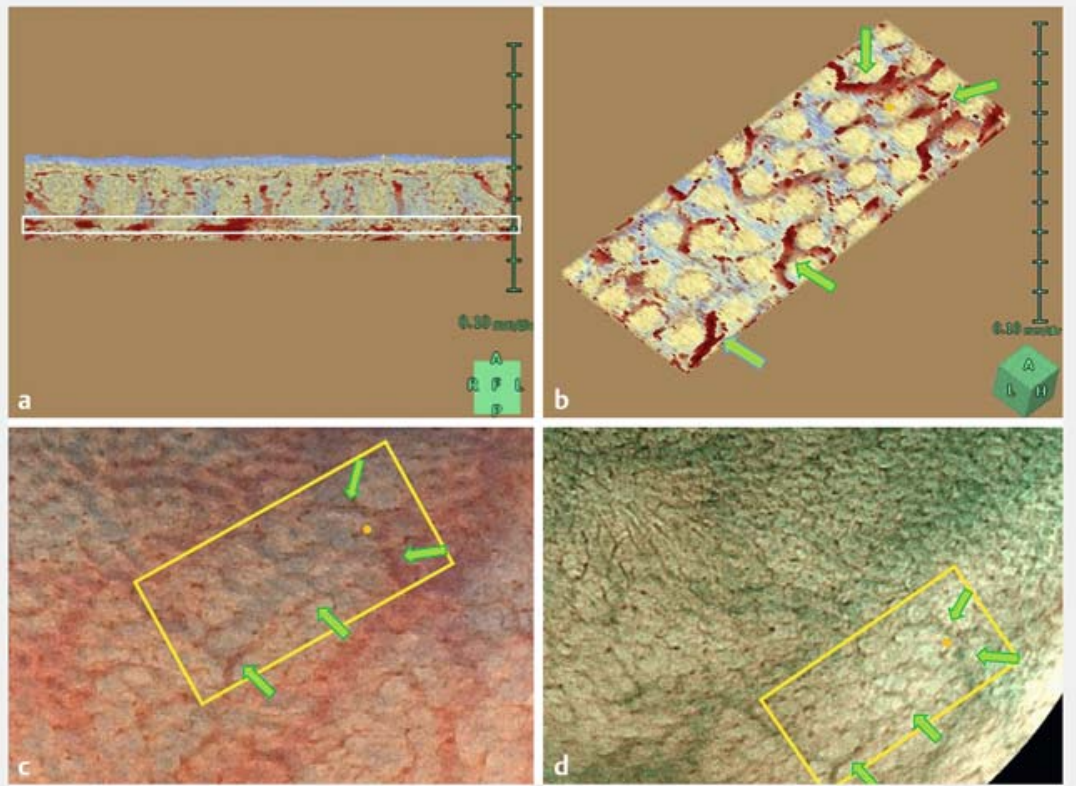

- Fig. 1 Comparison between: a, b the histological images obtained with digital microscopy and; $\mathbf{c}, \mathbf{d}$ the horizontal plane endoscopic images showing: a in the 2D reconstructed image of the colonic mucosa viewed from the lateral side, the deep mucosal capillary plexus (white rectangle), which exists at a depth of approximately $200 \mu \mathrm{m}$ from the surface; $\mathbf{b}$ in the reconstructed horizontal $3 \mathrm{D}$ image, the capillary plexus in the deep mucosa; $\mathbf{c}$ the same area on low magnification endoscopic view after indigo carmine staining; $\mathbf{d}$ the same area on low magnification imaging with blue light imaging (BLI). The yellow squares show the same area, the green arrows show identical capillaries, and the orange dots show the identical pit. 
scopically observable capillary plexus in the deep colonic mucosa.

Endoscopy_UCTN_Code_CCL_1AD_2AJ

\section{Competing interests}

This research was supported financially by FUJIFILM Corporation. Yuichi Teramura is an employee of FUJIFILM Corporation. The remaining authors declare that they have no conflict of interest.

\section{The authors}

Fumikazu Koyama ${ }^{1,2}{ }^{\odot}$, Takeshi Ueda ${ }^{3}{ }^{\ominus}$, Kohei Morita $^{4}$, Hisao Fujii ${ }^{5}$, Yuichi Teramura ${ }^{6}$, Chiho Obayashi $^{4}$, Masayuki Sho ${ }^{1}$

1 Department of Surgery, Nara Medical University, Kashihara, Japan

2 Division of Endoscopy, Nara Medical University Hospital, Kashihara, Japan

3 Department of Surgery, Minami-Nara General Medical Center, Yoshino, Japan

4 Department of Diagnostic Pathology, Nara Medical University, Kashihara, Japan

5 Department of Gastrointestinal Endoscopy and IBD Center, Heiwakai Yoshida Hospital, Nara, Japan
6 Endoscopy Systems Division, Medical Systems Business Division, FUJIFILM

Corporation, Tokyo, Japan

Corresponding author

\section{Fumikazu Koyama, MD}

Department of Surgery, Division of Endoscopy, Nara Medical University, 840 Shijo-cho, Kashihara-city, Nara, 634-8522, Japan

fkoyama@naramed-u.ac.jp

\section{References}

[1] Ohshima S. Volume analyzer SYNAPSE VINCENT for liver analysis. J Hepatobiliary Pancreat Sci 2014; 21: 235-238

[2] Ueda T, Morita K, Koyama F et al. A detailed comparison between the endoscopic images using blue laser imaging and three-dimensional reconstructed pathological images of colonic lesions. PLos One 2020; 15 : e0235279

[3] Skinner SA, O'Brien PE. The microvascular structure of the normal colon in rats and humans. J Surg Res 1996; 61: 482-490

[4] Kachlik D, Baca V, Stingl]. The spatial arrangement of the human large intestinal wall blood circulation. J Anat 2010; 216: 335-343
Bibliography

Endoscopy 2021; 53: E417-E418

DOI $10.1055 / a-1324-2429$

ISSN 0013-726X

published online 27.1.2021

(c) 2021. Thieme. All rights reserved.

Georg Thieme Verlag KG, Rüdigerstraße 14,

70469 Stuttgart, Germany

\section{ENDOSCOPY E-VIDEOS}

https://eref.thieme.de/e-videos

口回 Endoscopy E-Videos is a free

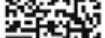
access online section, reporting on interesting cases and new

techniques in gastroenterological endoscopy. All papers include a high quality video and all contributions are freely accessible online.

This section has its own submission website at https://mc.manuscriptcentral.com/e-videos 